\title{
LA INFLUENCIA DE LOS RECURSOS AUDIOVISUALES PARA EL APRENDIZAJE AUTÓNOMO EN EL AULA
}

\author{
The Influence of Audiovisual Resources for Autonomous Learning in the \\ Classroom
}

A influência dos recursos audiovisuais para a aprendizagem autónoma na aula

\author{
María Marcos Ramos, Universidad de Salamanca (España) \\ mariamarcos@usal.es
}

Miguel Moreno Méndez, Universidad de Salamanca (España)

miguel.moreno@usal.es

Recibido: 5 de octubre de 2018

Aprobado: 4 de marzo de 2019

\section{RESUMEN}

El rápido avance de las nuevas tecnologías y de las dinámicas económicas ha sembrado una gran incertidumbre sobre a qué retos habrá de enfrentarse la sociedad. Ello incrementa la importancia del aprendizaje autónomo al favorecer la adaptación constante a lo largo de la vida. Debido al auge del consumo de contenidos audiovisuales entre los jóvenes, el empleo de estos contenidos como recursos didácticos en el aula puede ser una herramienta eficaz para comprender conceptos y fomentar su aprendizaje autónomo. Esto potenciará la capacidad de Aprender $a$ aprender del alumnado para conseguir en el futuro una mayor autonomía y adaptación laboral. 
En este artículo se muestran los resultados más significativos de un cuasi experimento realizado a cuarenta y siete alumnos/as de $3^{\circ}$ de Eso que buscaba conocer si el uso de recursos audiovisuales en el aula era relevante para su aprendizaje. Uno de los principales objetivos era hallar la utilidad didáctica que pueden tener estos recursos, la cual aumentó de manera significativa, según los datos del cuasi experimento, tras el visionado de un fragmento de la película Tiempos Modernos (Charles Chaplin, 1936). Entre las conclusiones, destaca que puede existir relación entre la percepción de utilidad de los contenidos audiovisuales y el nivel de aprendizaje autónomo del alumnado.

Palabras clave: alfabetización audiovisual, Aprender a aprender, aprendizaje autónomo, recurso didáctico, recursos audiovisuales.

\begin{abstract}
The fast progress of new technologies and the current economic dynamics have generated uncertainty about future challenges. This situation increases the relevance of autonomous learning, given that it benefits constant adaptation throughout life. The growth of the audiovisual content consumption, especially among teenagers, brings new opportunities. The use of this audiovisual content as a didactic resource might be useful to understand new concepts and improve autonomous learning. This approach will benefit students, improving learning to learn capacity. Through this method, labor adaptation and self-reliance are achievable.

This article shows the most significant results of a quasi-experiment carried out with 50 pupils of ninth grade to know if the use of the audiovisual resources in the classroom is relevant to learn the curricular concepts. Knowing the teaching effectivity of these resources was one of the main objectives, which improved in a significant way, in accordance with the data gathered in the test, after watching a passage of the film Modern Times. Among the conclusions, the relationship between the perception of audiovisual content and its utility and how students relate this with their learning to learn abilities stands out.
\end{abstract}

Keywords: Audiovisual resources, autonomous learning, lifelong learning, media literacy, teaching resource.

\title{
RESUMO
}

O rápido avanço das novas tecnologias e das dinâmicas econômicas têm semeado uma grande incerteza sobre a que desafios terá de enfrentar a sociedade. Isso incrementa a importância da aprendizagem autônoma ao favorecer a adaptação constante ao longo da vida. Devido ao auge do consumo de conteúdos audiovisuais entre os jovens, o emprego destes conteúdos como recursos didáticos na aula pode ser uma ferramenta eficaz para compreender conceitos e fomentar sua aprendizagem autônoma. Isto potenciará a capacidade de aprender a aprender dos alunos para conseguir no futuro uma maior autonomia e adaptação laboral.

Neste artigo se mostram os resultados mais significativos de uma quase-experimento realizado a 47 alunos/as de $3^{\circ}$ de ESO (Escola Secundária Obrigatória) que buscava conhecer se o uso de recursos audiovisuais na aula era 
relevante para sua aprendizagem. Um dos principais objetivos era encontrar a utilidade didática que podem ter estes recursos, a qual melhorou de maneira significativa, segundos dados do quase-experimento, após o visionado de um fragmento da película Tempos Modernos (Charles Chaplin, 1936). Entre as conclusões, destaca-se que pode existir relação entre a percepção de utilidade dos conteúdos audiovisuais e o nível de aprender a aprender dos alunos.

Palavras-chave: Alfabetização audiovisual; aprender a aprender; aprendizagem autônomo; recurso didático; recursos audiovisuais

\section{Introducción}

El gran desarrollo tecnológico que se ha producido durante los últimos años, junto con la globalización, presenta un incierto futuro en el ámbito académico y laboral. Una incertidumbre que plantea nuevos retos curriculares en las instituciones educativas: ¿qué conocimientos y competencias deben trabajarse con el alumnado actualmente? Un desafío que no es nuevo y que ya se planteaba hace años:

No sólo los educadores se enfrentan con situaciones inéditas [...]; los mismos alumnos están sujetos a idéntica situación de permanente cambio y [...] se acentuará en el futuro [...]. Por esto se alerta repetidamente que el hombre del futuro [...] o habrá adquirido la aptitud y la autodisciplina para aprender por sí mismo, o aprenderá muy poco (Mallas, 1997, p. 28).

Ante este futuro es fundamental que se trabaje con el alumnado en la autoevaluación de los resultados y conocimientos alcanzados para, así, tratar de cumplir una de las misiones principales de la enseñanza: dotar de las herramientas suficientes al aprendiz para que construya su propio conocimiento y, finalmente, su futuro. En la legislación española el aprendizaje autónomo se encuentra recogido en la competencia clave Aprender a aprender (Delors, 1997). Esta autonomía es fundamental en las teorías constructivas del aprendizaje bajo las cuales se han fundamentado los sistemas educativos occidentales con el proyecto DeSeCo, en el que se basa el currículum por competencias español:

Esta competencia [Aprender a aprender] se caracteriza por la habilidad para iniciar, organizar y persistir en el aprendizaje. Esto exige [...] la capacidad para motivarse por aprender [que] depende de que se genere la curiosidad y la necesidad de aprender, de que el estudiante se sienta protagonista del proceso y del resultado de su aprendizaje y, finalmente, de que llegue a alcanzar las metas de aprendizaje propuestas (Orden ECD/65/2015, p. 11).

Sin embargo, tradicionalmente, las investigaciones sobre el aprendizaje autónomo se han centrado principalmente en las técnicas de aprendizaje y su influencia en la enseñanza. La autonomía del estudiante se restringía, principalmente, a la elección de métodos para el estudio y a la personalización de los conocimientos. En este sentido, "los proyectos se han centrado fundamentalmente en las técnicas de estudio, las estrategias de aprendizaje y el 


\section{DISERTACIONES}

AVANCES

metaconocimiento" (Martín \& Moreno, 2007, p. 55). No obstante, en los últimos años se ha comenzado a investigar más sobre aspectos destacados en los procesos de enseñanza-aprendizaje, como la evaluación o los recursos, que influyen y son relevantes para la autonomía en el aprendizaje del estudiante y su futuro ciudadano. Entre estas investigaciones sobresale la realizada por Barak, Ashkar y Dori (2011), en la que no solo se hace hincapié en el aprendizaje con recursos audiovisuales, sino en la influencia de estos en la motivación del estudiante para aprender, o la de Barnett y Kafka (2007) en la que se incide en la conciencia del alumnado y en la influencia de los recursos audiovisuales en su aprendizaje.

Además, considerando la actual situación socio-económica y el rápido progreso tecnológico del mundo, la formación en Tecnologías de la Información y la Comunicación (TIC) parece vital, y más a tenor del gran consumo de nuevas tecnologías por la sociedad. Esta formación, incluida en la Competencia digital, es clave en la legislación y está regulada en la Ley Orgánica 8/2013 de 9 de diciembre para la mejora de la calidad educativa (LoMCE). Según dicha ley, la Competencia digital no solo debe promoverse para que los estudiantes adquieran un gran manejo de las nuevas tecnologías, sino para que comprendan los riesgos, los derechos y la responsabilidad que su empleo supone; esto es, trabajar los contenidos curriculares de tipo conceptual (técnica) y actitudinal (pensamiento crítico y ciudadano).

Para trabajar ambas competencias y enseñar los mismos contenidos curriculares, los recursos audiovisuales pueden ser una útil e innovadora alternativa debido al creciente consumo, especialmente entre los jóvenes, de productos audiovisuales, como películas, series, videojuegos o vídeos de Youtube. Ello reduciría, también, la brecha entre las actividades escolares y extra-académicas de los estudiantes y posibilitaría un refuerzo fuera de las aulas. Para esto, sin embargo, se necesita el esfuerzo de los principales agentes sociales al respecto, como la familia, las instituciones educativas y los medios, como se indica en la Declaración de Grünwald:

La educación relativa a los medios de comunicación será más eficaz si los padres, los maestros, el personal

de los medios de comunicación y los responsables de las decisiones reconocen que todos ellos tienen un cometido que desempeñar en la creación de una conciencia crítica más aguda (Unesco, 1982, p. 1).

Por tanto, el uso de contenidos audiovisuales como recurso didáctico ofrece la posibilidad de trabajar el aprendizaje autónomo del alumnado, de formarles en el uso de las tic y de incrementar el interés de los estudiantes por los contenidos curriculares, tanto conceptuales como actitudinales. Un ejemplo es la investigación experimental realizada por Gilliam y Seltzer (1989) para conocer la influencia de una película en el conocimiento y la actitud de los estudiantes sobre VIH, si bien "las diferencias entre los dos grupos [el que había visto la película sobre viH y el que no], en cuanto a conocimiento y actitud, fueron escasas”. De hecho, por la estrecha relación entre los jóvenes y los contenidos audiovisuales, practicar estas estrategias pedagógicas puede favorecer incluso el repaso de estos contenidos durante su tiempo de ocio. Además, "la familiaridad de los estudiantes con los textos mediáticos convierte a estos textos en vehículos ideales para el análisis de cómo funciona el lenguaje visual” (Quin \& Sánchez Martínez, 1999, pp. 137-138).

\section{Estado de la cuestión}

El rápido crecimiento de los contenidos y la mejora en la usabilidad de la web ha abierto nuevas formas de consumo mediante el ordenador y, más concretamente, a través de Internet. Este desarrollo tecnológico en torno a la 


\section{DISERTACIONES}

AVANCES

Red generó, y sigue generando, un incremento del consumo de productos o contenidos audiovisuales en los últimos años especialmente entre niños y adolescentes, con los avances de la Web 2.0 y la opción de subir contenidos audiovisuales de gran tamaño y calidad a Internet. De hecho, según la Vigésima Encuesta aimc a usuarios de Internet - Navegantes en la red ${ }^{1}$ (realizada entre octubre y diciembre de 2017), un 48,6 \% de los encuestados afirma haber visto películas o series a través de Internet (sin descargar) durante los últimos treinta días. Un consumo y carga o descarga de esta clase de contenidos que puede realizarse bien, por ejemplo, mediante plataformas de contenidos, como Youtube ${ }^{2}$, o bien mediante webs que alojan vídeos o productos audiovisuales (desde páginas web de televisión hasta otra de compartición de contenidos entre usuarios). Así, "la multimedialidad [...] se ha convertido en el contenedor de todos los formatos de información, provocando que el usuario de Internet la demande cada días más" (Marín, 2006, p. 182).

No obstante, no solo sucede con la información, sino también con el entretenimiento, a tenor de los hábitos de consumo en la actualidad de la sociedad, puesto que "la visualización de programas, series y noticias en Internet son cada vez más frecuentes, especialmente entre los segmentos más jóvenes de la sociedad" (López, González \& Medina, 2011, p. 100).

Este incremento en el consumo de Internet, de nuevas tecnologías y de contenidos audiovisuales por parte de los jóvenes, y a edades cada vez más tempranas, hace prever que la sociedad del futuro tenga un comportamiento distinto al de anteriores generaciones, lo que acarreará una gran influencia en la cultura, los hábitos y los procesos de aprendizaje de los alumnos de hoy y mañana. Por ello, es necesario incluir esta clase de contenidos en el currículum educativo.

\section{El auge del consumo audiovisual entre adolescentes}

En los últimos años el visionado de contenidos en Internet en España se ha convertido en un hábito para la mayoría de los jóvenes. Según datos del informe La Sociedad de la Información en España 2016 (siE[16), realizado por la Fundación Telefónica, un 72,4 \% de los usuarios de Internet en España consume vídeos de sitios para compartir (como Youtube) y entre los jóvenes (de entre 16 y 24 años) el consumo de estos contenidos alcanza el 92,5\%. Nuevas formas y hábitos de consumo que han hecho que el cómo, el dónde y el cuándo se consume determinado producto audiovisual, y el mismo producto, haya variado. Un dato ejemplificador de esta creciente tendencia es el consumo de canales de televisión emitidos por Internet, actividad que ya realiza, según datos del Instituto Nacional de Estadística (INE) recogidos en siE[16, el 44,3\% de los usuarios españoles en 2016, así como el visionado de películas o vídeos bajo demanda.

1 El estudio fue realizado por la Asociación para la Investigación de Medios de Comunicación (AImc), titulado Vigésima encuesta aimc a usuarios de Internet - Navegantes en la red, en el que la muestra total de entrevistados fue de 15252 individuos.

2 Un 81,3\% de las personas que participaron en la encuesta Encuesta AImc afirman haber visualizado online vídeos "tipo Youtube” durante los últimos treinta días. 


\section{DISERTACIONES}

AVANCES

No obstante, este consumo no sólo es propio de los nativos digitales (Prensky, 2001), sino también de los millennials (Howe \& Strauss, 2000). Este predominio de Internet en el entretenimiento de los jóvenes se debe, entre otras razones, a que "pueden elegir lo que ven y escuchan cuando quieren [...] por lo que no les hace falta sintonizar el aparato televisivo y compartir sus momentos de ocio con otras personas si no lo desean" (López et al., 2011). En este aspecto, los jóvenes son usuarios "cada vez más propensos a un consumo mediático autónomo e independiente respecto al resto de su familia” (Fedele \& García-Muñoz, 2010, p. 53). Se trata del fenómeno conocido como bedroom culture (Bovill \& Livingstone, 2001) el cual se ha visto favorecido por la presencia de Internet o dispositivos digitales en los dormitorios infantiles, conocidos como media-rich child's bedrooms, lo que promueve su consumo desde niños.

\section{La enseñanza por competencias}

El actual sistema educativo español se basa en la enseñanza por competencias, cuyo objetivo es que el alumnado adquiera las herramientas cognitivas necesarias para su posterior desarrollo en el entorno académico, laboral y social. Este nuevo método trata de acercar la realidad cotidiana a los estudiantes para que se enfrenten satisfactoriamente a los desafíos sociales y laborales del futuro.

Tradicionalmente, el objetivo principal de la educación era la perduración de los contenidos seleccionados por el profesor en los estudiantes mediante la memorización. Un método ligado a las teorías asociativas, fundamentales para la adquisición de información y para poder recordarla, pero no tanto para añadir nuevos conocimientos. Se trata, pues, de una fórmula opuesta a las teorías constructivistas que principalmente desarrollaron Piaget (1987) y Vigotski (1989), basadas en la comprensión de los conceptos a aprender y la construcción personal del propio conocimiento; es decir, "que en todos sus niveles, la inteligencia es una asimilación de lo dado a estructuras de transformaciones [...], y que estas estructuras consisten en organizar lo real, en acto o pensamiento, y no simplemente copiarlo" (Piaget, 1987, p. 39).

De la misma forma, estas competencias incluyen diferentes tipos de aprendizaje que se basan en los definidos por Delors en su célebre informe titulado Los cuatro pilares de la educación (1997): 'aprender a conocer'; 'aprender a hacer'; 'aprender a vivir juntos, aprender a vivir con los demás' y 'aprender a ser', que inciden en los conocimientos epistemológicos per sé, su aplicación profesional y social y su incidencia ciudadana.

En la legislación española es la Lomce la que establece las competencias en el sistema educativo español. Esta contempla siete competencias clave, pero se destacará la competencia Aprender a aprender al ser la que atañe directamente al tema de estudio de esta investigación.

\section{La competencia Aprender a aprender}

En detrimento de la enseñanza tradicional de técnicas de estudio y de la transmisión del conocimiento del profesorado a los alumnos, en la actualidad se ha comenzado a dar mayor relevancia al trabajo sobre la autonomía del estudiante. Es decir, a ofrecer al alumnado todas las herramientas posibles para potenciar su autonomía: desde los distintos tipos de saber (conocimientos, destrezas y valores) a los propios elementos curriculares (metodología, recursos, evaluación, etc.). 


\section{DISERTACIONES}

AVANCES

Tecnologías y métodos computacionales para la investigación en ciencias sociales y comunicación

ISSN: 1856-9536

Doi: https://doi.org/10.12804/revistas.urosario.edu.co/disertaciones/a.7310

Volumen 13, Número 1 / Enero-junio 2020

Versión PDF para imprimir desde

http://revistas.urosario.edu.co/index.php/disertaciones

Como señalan Monereo et al. (2001) "más que enseñar contenidos debemos enseñar a los alumnos aquello que les permitirá, en un futuro próximo, ser capaces de aprender esos contenidos por sí mismos" (p. 5). Pero el objetivo no solo es "que dirijan por sí mismos su aprendizaje, sino también [...] que juzguen por sí mismo sobre sus resultados, [...] el autocontrol del aprendizaje autónomo" (Aebli, 1991, p. 158). Es decir, aprender de los errores y conocer por qué se han podido cometer para no volver a repetirlos.

Aprender a aprender implica mucho más que memorizar y retener nociones, pues el estudiante debe investigar, comunicarse e interactuar. En este escenario deja de adquirir gravitación prevalente el volumen de contenidos [...] y ocupa, en cambio, un papel decisivo el modo en que son entregados (Kaplún, 2002, p. 236).

Como indica la Ley Orgánica 2/2006 de 3 de mayo sobre Educación (LOE), recogida en el Real Decreto 1631/2006, de 29 de diciembre, por el que se establecen las enseñanzas mínimas correspondientes a la Educación Secundaria Obligatoria, Aprender a aprender "significa ser consciente de lo que se sabe y de lo que es necesario aprender, de cómo se aprende, y de cómo se gestionan [...] de forma eficaz los procesos de aprendizaje” (p. 25).

Es por ello que actividades como la auto-evaluación o la evaluación entre iguales pueden ser muy positivas, ya que así el estudiante puede aprender de sus errores y ser consciente de su progreso. Sin embargo, esta autonomía requiere de un trabajo continuo con un mediador en este proceso de enseñanza-aprendizaje:

Aunque el objetivo es conseguir que finalmente el alumno pueda llegar a evaluar cuándo aprende y cuándo no y de lo que hace qué es lo que más le ayuda a aprender, esta autonomía no llegaría a alcanzarse si no fuera porque el profesor controla durante mucho tiempo el conjunto de la actividad [...]. Es un control que tiene como objetivo traspasarse, es decir, dejar de ser ejercido por el docente porque ya es capaz de llevarlo a cabo el estudiante (Martín \& Moreno, 2007, p. 116).

\section{La alfabetización audiovisual}

El creciente consumo de vídeos y contenidos audiovisuales entre la sociedad, especialmente entre los jóvenes, ha provocado que emerja una pregunta: ¿cuán importante es educar a las nuevas generaciones en medios de comunicación y con formatos audiovisuales?

Ya hace décadas se cuestionaba, como en la Declaración de Grünwald (1982), la relevancia que podían tener los medios de comunicación en la educación para lograr la alfabetización digital o mediática y, así, "enseñar a niños y a adolescentes cómo leer imágenes, cómo interpretarlas, cómo interactuar con los medios de comunicación, con las nuevas tecnologías..." (Marcos, 2010, p. 6). Por tanto, introducir contenidos, recursos y estrategias en los centros educativos vinculados a la alfabetización audiovisual es clave para la formación mediática de los jóvenes, pero también para lograr una mayor eficacia de los recursos audiovisuales y tecnológicos en el aula:

La alfabetización informacional se encuentra en el corazón mismo del aprendizaje a lo largo de la vida. Capa-

cita a la gente de toda clase y condición para buscar, evaluar, utilizar y crear información eficazmente para

conseguir sus metas personales, sociales, ocupacionales y educativas (Declaración de Alejandría, 2005).

Se hace así imprescindible reflexionar acerca de la importancia de la alfabetización audiovisual del alumnado de Primaria y Secundaria, no solo por la relevancia que actualmente ya cobran los contenidos audiovisuales y mediáticos, sino por su influencia en el futuro a medida que los jóvenes de hoy se incorporen a la vida adulta. A pesar de todo, la desconfianza sobre lo audiovisual persiste, lo que priva al alumnado de una completa comprensión de los 


\section{DISERTACIONES}

AVANCES

Tecnologías y métodos computacionales para la investigación en ciencias sociales y comunicación

ISSN: 1856-9536

Doi: https://doi.org/10.12804/revistas.urosario.edu.co/disertaciones/a.7310

Volumen 13, Número 1 / Enero-junio 2020

Versión PDF para imprimir desde

http://revistas.urosario.edu.co/index.php/disertaciones

contenidos: "Los educadores no debemos centrar nuestra tarea en preservar a los alumnos de las influencia de la imagen; nuestro reto consiste en ser capaces de convertirlos en auténticos comunicadores [...] críticos y 'pensantes', inteligentes, libres" (García \& Benítez, 1998, p. 206).

Es en este punto donde radica la importancia del profesorado como mediador del aprendizaje: se deben dar a conocer todas las herramientas posibles al aprendiz y trabajarlas con él para que, finalmente, logre su autonomía también mediante las nuevas tecnologías y los recursos audiovisuales (que entre otros beneficios puede promover un sentido crítico y un aprendizaje integrado).

\section{El uso de los recursos audiovisuales en los procesos de enseñanza-aprendizaje}

En la actualidad, los jóvenes se encuentran más ligados que nunca a los recursos audiovisuales, a tenor del tiempo que dedican a su consumo, de la proliferación de formatos y del manejo de las nuevas tecnologías. Es por este motivo que las series o videojuegos, por ejemplo, ya se contemplan para ser empleados como recurso didáctico y su consumo con fines educativos puede ser llevado a cabo fuera del horario escolar; hechos sin duda novedosos respecto a anteriores décadas.

No obstante, el empleo de los instrumentos o medios y materiales didácticos está precedido de una estrategia educativa, por lo que hablamos de recurso/s audiovisual/es al estar englobado/s dentro de estos instrumentos y de la necesaria estrategia didáctica previa. Así, se alejan de la llamada educación bancaria, dado que "los medios audiovisuales, en la educación tradicional, se usan solamente como refuerzos para la transmisión de los contenidos. Es educación 'envasada'” (Kaplún, 2002, p. 25).

Se hace así necesario que el profesorado tenga un cierto nivel de conocimiento de narrativa y lenguaje audiovisual y de manejo de las TIC, que le permita ejercer este papel alfabetizador para el aprendizaje de sus estudiantes y, de este modo, combatir la posible mala influencia que puedan generar en los jóvenes: "Para bien o para mal, los medios de comunicación son uno de los grandes educadores, esculturizadores, de los niños y adolescentes -incluso de los adultos-. Por eso, debemos hacer que sean para bien, que se utilicen de manera constructiva" (Marcos, 2010, p. 73).

El conocimiento para el empleo de estos recursos y la proactividad del alumnado son, por tanto, indispensables para trabajar la competencia Aprender a aprender mediante recursos audiovisuales. La actividad no era para Vigotski (1989) respuesta o reflejo solamente, "sino que implicaba un componente de transformación del medio con ayuda de instrumentos. El concepto de actividad estaba muy relacionado con el de mediación" (Rivière, 1988, p. 41). No obstante, esta mediación - dirigida o no por el profesorado- puede realizarse a través de instrumentos o recursos, pudiéndose utilizar desde películas o videojuegos a plataformas educativas web. Esta es la importancia del consumo de estos productos audiovisuales, ya que sin un mediador sí puede existir mediación (trabajando la competencia Aprender a aprender) y, por tanto, refuerzo educativo que sirva de ayuda para el aprendizaje:

Ayudar a los jóvenes a convertirse en buenos aprendices puede significar atreverse a abandonar la creencia

de que la más alta responsabilidad de un enseñante es ser omnisciente (pues si de lo que se trata es de conseguir conocimiento, normalmente es más fácil adquirirlo a través de Internet) (Claxton, 2005, p. 263).

Ello puede significar, por ejemplo, que un joven alumno aficionado a las películas o los videojuegos puede aprender o relacionar conceptos tratados en clase mientras disfruta de su consumo. Además, habrá más posibilidades de 


\section{DISERTACIONES}

AVANCES

Tecnologías y métodos computacionales para la investigación en ciencias sociales y comunicación

ISSN: $1856-9536$

Doi: https://doi.org/10.12804/revistas.urosario.edu.co/disertaciones/a.7310

Volumen 13, Número 1 / Enero-junio 2020

Versión PDF para imprimir desde

http://revistas.urosario.edu.co/index.php/disertaciones

que el aprendizaje se produzca cuantas más herramientas se le hayan facilitado en el aula y más se haya trabajado con estos materiales con un mediador; en este caso, el docente. Es decir, cuando se haya llevado a cabo la estrategia de edu-entretenimiento que "es el proceso de diseñar e implementar un mensaje mediático, tanto para entretener como para educar, con el objetivo de incrementar el conocimiento de la audiencia sobre un problema educativo, crear una actitud favorable y cambiar su comportamiento" (Singhal \& Rogers, 1999, p. 267). Otros autores resaltan, también, este aspecto y la influencia que puede tener para "mejorar y facilitar diferentes etapas del comportamiento y del cambio en favor de la sociedad” (Bouman, 1999, p. 25). El edu-entretenimiento supone, por tanto, un acercamiento del aprendiz a los conceptos educativos a adquirir, a través de los medios y el entretenimiento, y de sus intereses. Así, habrá una mayor cercanía a los conceptos cuanto mayor interés susciten en el aprendiz y se reducirá una posible actitud desfavorable, lo que a su vez posibilitaría una mayor predisposición al aprendizaje.

Este mayor interés puede generar un mayor grado de aprendizaje en el estudiante, al haber una actitud más favorable. Incluso podría suceder que los contenidos y medios de entretenimiento puedan ser mediadores del aprendizaje de conocimientos por sí mismos, si, finalmente, así son percibidos por los aprendices mediante la práctica del edu-entretenimiento, al mismo tiempo que de ellos obtienen entretenimiento u ocio.

En los últimos años ha crecido el número de investigaciones relacionadas acerca de la utilidad de los medios audiovisuales (principalmente películas) para facilitar o mejorar el aprendizaje de conceptos curriculares o educativos en las aulas. También se ha estudiado su capacidad de proporcionar entretenimiento a los alumnos -cuestión relacionada con la anterior utilidad, al poder incrementar la predisposición al aprendizaje- $\mathrm{y}$ otros valores relacionados con la educación, como la motivación, relación con la vida diaria o relevancia (presente y futura) de los contenidos educativos. En este ámbito, cabe citar algunas investigaciones destacadas, como la realizada por Barak, Ashkar y Dori en 2011, en la que trataban de conocer el efecto de las películas animadas sobre las mejoras en el aprendizaje en el campo de la ciencia, de estudiantes de Primaria y su motivación para aprender. ${ }^{3}$

El uso de fragmentos de películas fue el recurso audiovisual empleado por Barnett y Kafka (2007) para analizar la influencia de los recursos audiovisuales sobre el conocimiento científico de los alumnos para mejorar su capacidad crítica y de análisis sobre la ciencia. En las evaluaciones de final de curso los estudiantes indicaron la importancia de los fragmentos de película empleados en el mantenimiento de su interés y en la ayuda para comprender mejor los conceptos científicos.

En niveles educativos superiores, como el universitario, Gilliam y Seltzer (1989) realizaron un experimento para evaluar si el visionado de una película incrementaba el conocimiento y la actitud de los estudiantes sobre viH, así como su actitud sobre personas homosexuales, y concluyeron que "el visionado de una película educacional, por su empleo en el experimento, parece ser insuficiente para educar a los estudiantes sobre este problema" (Gilliam \& Seltzer, 1989, p. 263).

3 En el artículo "Learning science via animated movies: its effect on students' thinking and motivation", exponen que su investigación fue experimental, con dos pre-test y post-test, con una población de 1335 alumnos de diferentes colegios. Los resultados indicaron que el uso de películas animadas mejora la capacidad explicativa y de entendimiento de conceptos científicos, además de que "el uso de películas animadas, sobre aquellos estudiantes que estudiaron ciencias a través de ellas, mejoró la motivación para aprender ciencia en términos de auto-eficacia, interés y disfrute, relación con la vida diaria e importancia para su futuro" (Barak, Ashkar \& Dori, 2011, p. 844). 


\section{DISERTACIONES}

AVANCES

Otras investigaciones también hacen hincapié en la relevancia de los posibles errores o inexactitudes que se dan en las películas. Un ejemplo es la de Marsh, Butler y Umanath (2012) cuyo objetivo era conocer cómo el uso de películas históricas para practicar el pensamiento crítico - a través de la identificación de inexactitudes históricas- podía afectar la adquisición de conocimiento histórico falso o real. La investigación concluyó que los estudiantes deberían recibir más retroalimentación de los profesores tras realizar estos ejercicios de pensamiento crítico, debido a que cuando ellos lo recibían, los malentendidos eran significativamente menos.

\section{Metodología}

A pesar de todo, todavía son escasas las investigaciones de campo que tratan de conocer la utilidad de los recursos audiovisuales para mejorar el conocimiento de los estudiantes en relación con su aprendizaje autónomo. De las investigaciones mencionadas, las realizadas por Barnett y Kafka (2007) o Barak, Ashkar y Dori (2011) son las que aluden directamente a la capacidad autónoma del estudiante.

Por ello, ante la escasez de investigaciones que analicen la influencia de los recursos audiovisuales para el aprendizaje, especialmente en España y en la etapa educativa de Secundaria y, además, la utilidad de estos recursos en el aula para trabajar el aprendizaje autónomo del alumnado, se decidió llevar a cabo una investigación experimental con el fin de indagar esta cuestión mediante la realización de trabajo de campo. Así, los objetivos a destacar son:

- Analizar si el uso de recursos audiovisuales en el aula ayuda al aprendizaje de contenidos, tanto conceptuales como actitudinales.

- Comprobar si el empleo de recursos audiovisuales favorece la percepción sobre su utilidad didáctica y, por tanto, mejora la capacidad para el aprendizaje autónomo respecto a los recursos a emplear.

En definitiva, el núcleo de la investigación yacía en analizar hasta qué punto los recursos audiovisuales pueden ser útiles para el aprendizaje del alumnado a largo plazo. Es decir, que los recursos dejen una cierta impronta en ellos a través de la experiencia y les haga, poco a poco, ser conscientes de los métodos, técnicas o recursos que les son útiles para aprender significativamente y cuáles son los motivos básicos para Aprender a aprender. Así, las hipótesis para cumplir los objetivos mencionados son:

- Hipótesis 1: El uso de recursos audiovisuales en el aula ayuda al alumnado, según su propia percepción, al aprendizaje de contenidos conceptuales y actitudinales.

- Hipótesis 2: Existen diferencias significativas en la percepción del aprendizaje entre el alumnado sobre el que se han empleado recursos audiovisuales para la enseñanza de contenidos respecto a aquel sobre el que no se han usado estos recursos.

- Hipótesis 3: La utilidad que para el alumnado tienen las películas como recurso didáctico (para comprender los conceptos de los contenidos curriculares; relacionar los contenidos de varias asignaturas; relacionarlos con la vida cotidiana y generar interés sobre los contenidos de clase) aumenta una vez se ha empleado una película como recurso en el aula en comparación con la opinión previa a su uso. 


\section{DISERTACIONES}

AVANCES

\section{Diseño y procedimiento}

La investigación, realizada en el año 2017, tiene un carácter cuasi experimental, ya que "no existe un control absoluto de la situación experimental" (Igartua, 2006, p. 339) ya que la selección del alumnado no fue aleatoria. Sobre el diseño de la investigación, en primer lugar, se realizó una encuesta a todos los participantes para conocer, entre otras cuestiones, la utilidad que ellos mismos creen que puede tener esta clase de recursos en el aula para su aprendizaje. En el cuestionario había una pregunta que conformaba un primer pre-test que posteriormente sería comparado con el post-test.

Por ello, se puede afirmar que fue un diseño pre-test-post-test, con dos pre-test en dos tiempos diferentes: el primero, el día de la encuesta, y, el segundo, antes del visionado del recurso audiovisual. Los motivos fueron, además de las posibles limitaciones que se produjeran posteriormente, evitar en mayor medida que los estudiantes recordaran sus respuestas a cuestiones como la utilidad de los recursos audiovisuales (películas, videojuegos, etc.) para aprender conceptos de clase, generar interés en las asignaturas de clase y, además, obtener información adicional sobre sus intereses y hábitos con la tecnología y lo audiovisual.

Antes del inicio de la clase se proporcionó a los alumnos de los dos grupos un cuestionario, que constaba de preguntas con respuestas de escalas del 0 al 10, con el objetivo de medir si se habían obtenido resultados significativos mediante la realización del experimento. Estas cuestiones versaban sobre el nivel de interés y conocimientos sobre el tema (la industrialización y la producción en cadena), el conocimiento sobre la situación laboral de la época y su similitud con el presente y la influencia que puede tener el trabajo y las nuevas tecnologías sobre las personas.

Una vez completado este cuestionario, se explicó una serie de contenidos básicos a modo de contextualización y acercamiento al tema, con apoyo de dos fotografías, durante un tiempo de diez minutos, para, posteriormente, en el grupo experimental reproducir el recurso audiovisual y, en el grupo control, continuar con la clase rutinaria: la explicación de los conceptos que se tratan en el film de forma oral y con el apoyo de dos fotografías.

Tras la visualización del fragmento de película y de la explicación oral, se pidió que realizaran el segundo cuestionario, que comenzaba con las mismas preguntas en la misma serie de escalas (para medir su posible cambio) y, posteriormente, si la clase les había ayudado a comprender mejor o no cómo era aquella época o el concepto de producción en cadena, así como por qué creían que les había ayudado o por qué creían que no, de forma explicativa.

En el cuestionario del grupo experimental se añadieron, después de las preguntas comunes a ambos grupos, algunas vinculadas al recurso audiovisual, como qué emociones o sentimientos habían tenido cuando habían sabido que iban a ver una película en clase; si su interés cambió cuando supieron que la película era en blanco y negro; y si creen que las películas pueden ser útiles para el aprendizaje de los conceptos de clase, para relacionar los conceptos de varias materias entre sí o relacionarlos con la vida diaria (directamente relacionado con su capacidad de aprendizaje autónomo). De este modo, se buscaba establecer alguna relación entre las respuestas en la encuesta (primer pre-test) y las del cuestionario (post-test) tras ver el film.

Con el fin de evitar la posible contaminación entre los diferentes grupos, debido a que la clase se daría con un día de diferencia - por el horario escolar-, el grupo control fue el primero con el que se hizo la clase al ser el que menos novedades - susceptibles de ser comentadas- ofrecía en comparación con el experimento. 


\section{DISERTACIONES}

AVANCES

\section{Participantes}

La muestra de la investigación estuvo compuesta por 47 participantes, alumnos/as del curso de $3^{\circ}$ de Eso, de los que 29 eran hombres (61,7\%) y 18 eran mujeres (38,3\%). En cuanto a la edad, 28 tenían 14 años cuando realizaron la encuesta (59,6 \%); 17 tenían 15 años (36,2\%) y 2 tenían 16 años (4,2\%), por lo que la media de edad de los participantes es 14.45 (DT=0,58).

En el experimento se midió si el visionado de un recurso audiovisual (un fragmento) ${ }^{4}$ facilitaba el aprendizaje de contenidos conceptuales y actitudinales y, también, si este visionado favorecía la percepción de los recursos audiovisuales como recurso didáctico útil. Para ello se compararon las puntuaciones de la clase que visionó la película y de la clase en la que no se utilizó. Así, finalmente, tomaron parte en el experimento un total de 27 alumnos: 13 participantes en el grupo control (de la clase de $3^{\circ}$ de ESO c) y 14 en el grupo experimental ( $3^{\circ}$ de ESO D), (toda la sección bilingüe). En cuanto al género, 15 eran alumnos (55,6 \%) y 12 alumnas (44,4\%). Sobre la edad, 18 tenían 14 años (66,7 \%), 8 tenían 15 años (29,6\%), y un alumno, 16 años (3,7 \%), por lo que la media de edad es $M=14,37$; $\mathrm{DT}=0,56$. A pesar de estas limitaciones mencionadas, el método de elección de las clases como grupo experimental y del grupo control fue aleatorio.

\section{Instrumentos y variables}

Los instrumentos empleados en el experimento fueron una encuesta, facilitada a la totalidad del alumnado de las clases - que contenía a su vez una variable que posteriormente serviría como un primer pre-test- y un cuestionario facilitado solo al alumnado de la sección bilingüe antes (segundo pre-test) y después del visionado de la película (post-test). ${ }^{5}$

Para la encuesta se emplearon escalas Likert en la mayoría de las preguntas, algunas de las cuales se incluyeron en el cuestionario para realizar el segundo pre-test y el post-test con el fin de conocer la percepción de los alumnos sobre la utilidad de las películas para comprender contenidos del currículum educativo; relacionar los

4 El fragmento seleccionado para ver en clase fue de la película Tiempos Modernos (Charles Chaplin, 1936) y comprendía desde el inicio de la película hasta el traslado al hospital del obrero (quince minutos), con el objetivo de una mayor validez en el experimento, puesto que en una clase de secundaria el tiempo es limitado y la película ejerce de recurso y apoyo a los contenidos. La selección de este film se debió a la unidad didáctica que se estaba tratando en ese momento en la asignatura, 'El sector secundario y la industrialización'. Además, en el film se realiza una descripción histórica y cercana de aquel contexto: cómo era la sociedad en el periodo de entreguerras - de Estados Unidos-y la situación económica, laboral e histórica, como la producción en cadena.

5 Las preguntas analizadas para conocer si el recurso audiovisual ha ayudado al aprendizaje de contenidos conceptuales fueron: "grado de conocimiento que tú crees tener EN ESTOS MOMENTOS sobre la producción en cadena" y "en qué medida crees EN ESTOS MOMENTOS que era duro el trabajo en la industria en la década de 1920". En cuanto a los contenidos actitudinales, las preguntas analizadas son "qué influencia crees EN ESTOS MOMENTOS que puede tener el trabajo para una persona en su salud mental" y "en qué medida crees EN ESTOS MOMENTOS que la tecnología mejora la vida diaria de las personas". 


\section{DISERTACIONES}

AVANCES

contenidos de diferentes asignaturas; relacionar estos contenidos con la realidad; y para generar interés sobre los mismos contenidos. De este modo, también se podría conocer las posibles diferencias en las respuestas del alumnado sobre estas variables antes y después de la impartición de la clase con el visionado de la película y, así, medir la influencia del recurso audiovisual. En el cuestionario también se emplearon escalas del 0 al 10 para una mayor concreción de las diferencias por el experimento y el menor número muestral.

Con el objeto de evitar posibles datos espurios, además de advertir al alumnado sobre la importancia de su respuesta sincera y la comprensión de todas las cuestiones que se planteaban, en las escalas del 0 al 10, se expuso el término 'Nada' en el margen izquierdo de la escala y al lado del número 0 y el término 'Mucho/a' en el lado contrario y al lado del número 10. Sin embargo, en las escalas Likert las variables se ordenaron - de izquierda a derecha- de 'Mucho' a 'Nada' y viceversa en los diferentes bloques que englobaban las cuestiones con el objetivo de lograr mayor fiabilidad.

\section{Resultados}

Con el fin de comparar los resultados entre el alumnado que había participado en el experimento, las pruebas que se realizaron fueron la $\mathrm{W}$ de Wilcoxon, para muestras relacionadas, y la $U$ de Mann-Whitney, para muestras independientes; pruebas no paramétricas debido al escaso número muestral en el experimento (trece y catorce casos en el grupo control y en el grupo experimental, respectivamente). El fundamento de estas pruebas es la comparación de las medianas entre grupos en lugar de la media, tal y como se hace con la prueba paramétrica t de Student. Se seleccionó la prueba $U$ de Mann-Whitney ya que ante el escaso número de casos podría no ser tan fiable usar la t de Student para conocer si existen diferencias significativas debido a la distorsión que podrían causar los datos extremos.

\section{Comprobación de la equivalencia de los grupos}

Antes del propio análisis de los datos se verificó que los grupos que participaron en el experimento fueran homogéneos en variables sociodemográficas básicas como el sexo o la edad. ${ }^{6}$ Esta prueba es fundamental para comprobar si existen diferencias significativas por razones de edad o sexo entre las dos clases, y, por eso, se realizó una prueba t de Student. Esta prueba mostró que no existen diferencias significativas $(p>0,05)$ por razones de sexo entre el grupo control $(M=1,45$, $D T=0,510)$ y el grupo experimental $(M=1,32, D T=0,476)$, si bien existe una mayor proporción de hombres que de mujeres. En cuanto a la edad, tampoco se encontraron diferencias significativas $(p>0,05)$ entre el grupo control $(M=14,55, D T=0,596)$ y el experimental $(M=14,36, D T=0,569)$.

6 "Si los grupos experimentales son homogéneos se podrá inferir con garantía que cualquier diferencia en las variables dependientes consideradas (...) se debe o se explica por el impacto provocado por la(s) variables(s) independiente(s) y no por otros factores o variables extrañas" (Igartua, 2007, p. 175). 


\section{DISERTACIONES}

AVANCES

\section{Hipótesis 1}

La primera de las hipótesis esperaba encontrar que, según la propia percepción del alumnado, el uso de recursos audiovisuales en el aula les ayudara a aprender contenidos conceptuales y actitudinales. Para comprobar si los recursos audiovisuales, con el ejemplo del visionado de la película, ayudan al aprendizaje de estos contenidos se compararon las respuestas ${ }^{2}$ del alumnado en el pre-test y el post-test que realizaron el día en que se utilizó el recurso, con el empleo de una escala del 0 al 10 donde 0 significa 'Nada' y 10 'Mucho'.

Los resultados que se obtuvieron, tras realizar la prueba de Wilcoxon debido al pequeño tamaño muestral (menos de treinta casos), en la pregunta sobre el grado de conocimiento de la producción en cadena ( $\mathrm{z}=-2,458$, $p<0,05)$ mostraron que hubo diferencias significativas: pre-test $(M d=4,00)$ y post-test $(M d=5,50)$; y en la pregunta sobre la dureza del trabajo en la industria del Periodo de entreguerras, las diferencias esta vez no fueron significativas $(z=-1,768, p>0,05)$, siendo $(M d=8,00)$ en el pre-test y $(M d=9,00)$ en el post-test. Por tanto, a tenor de los datos analizados, se encontró que los recursos audiovisuales ayudan al alumnado al aprendizaje de contenidos conceptuales, pero no de manera significativa.

Tabla 1. Influencia en el aprendizaje de contenidos conceptuales

\begin{tabular}{|l|c|c|c|}
\hline \multicolumn{1}{|c|}{ Variable } & Significatividad & Pre-test (Md) & Post-test (Md) \\
\hline Conocimiento de la producción en cadena & $p<0,05$ & 4,00 & 5,50 \\
\hline Dureza del trabajo en la industria la década de 1920 & $p>0,05$ & 8,00 & 9,00 \\
\hline
\end{tabular}

Fuente: elaboración propia.

También se realizó la prueba de Wilcoxon sobre los contenidos actitudinales, y los resultados a la pregunta acerca de la influencia del trabajo en la salud mental de una persona no reflejaron diferencias significativas ( $\mathrm{z}=-\mathbf{0}, 710$, $p>0,05)$ entre el pre-test $(M d=8,50)$ y el post-test $(M d=8,00)$. En el caso de la pregunta sobre cómo la tecnología mejora la vida de las personas, tampoco hubo diferencias significativas $(z=-0,351, p>0,05)$ : pre-test $(\mathrm{Md}=9,00)$ y post-test $(M d=9,00)$. Por lo tanto, no se encontró que los recursos audiovisuales puedan ayudar de manera significativa al aprendizaje de contenidos actitudinales.

No obstante, se debe mencionar que el margen de ayuda o influencia del recurso era mayor sobre el grado de conocimiento, pre-test ( $\mathrm{Md}=4,00)$, que respecto al resto de cuestiones cuyas medianas eran muchos más elevadas (se situaban, al menos, en 8 puntos en la escala del 0 al 10), lo cual reducía la posibilidad de influir significativamente sobre su aprendizaje. 


\section{DISERTACIONES}

\section{AVANCES}

Tecnologías y métodos computacionales para la investigación en ciencias sociales y comunicación ISSN: 1856-9536

Doi: https://doi.org/10.12804/revistas.urosario.edu.co/disertaciones/a.7310

Volumen 13, Número 1 / Enero-junio 2020

Versión PDF para imprimir desde

http://revistas.urosario.edu.co/index.php/disertaciones

Tabla 2. Influencia en el aprendizaje de contenidos actitudinales

\begin{tabular}{|l|c|c|c|}
\hline \multicolumn{1}{|c|}{ Variable } & Significatividad & Pre-test (Md) & Post-test (Md) \\
\hline Influencia del trabajo en la salud mental & $\mathrm{p}>0,05$ & 8,50 & 8,00 \\
\hline Mejora de la tecnología a la vida diaria & $\mathrm{p}>0,05$ & 9,00 & 9,00 \\
\hline
\end{tabular}

Fuente: elaboración propia.

\section{Hipótesis 2}

La segunda hipótesis esperaba que hubiera diferencias significativas en la percepción del aprendizaje entre aquel alumnado sobre el que se empleó el recurso audiovisual para la enseñanza de contenidos respecto a aquellos sobre los que no se utilizó.

Para comprobar si existen diferencias significativas entre el grupo control y el experimental, se llevó a cabo la prueba $U$ de Mann-Withney que compara la mediana de las respuestas entre ambos grupos. En este caso, la comparación se realizó sobre el cambio que se produjo entre el pre-test y el post-test a las respuestas de las preguntas anteriores. Así, acerca de la pregunta sobre el grado de conocimiento de la producción en cadena no se encontraron diferencias significativas $(z=-0,689, p>0,05)$ en el cambio producido entre el grupo control $(M d=2,00)$ y el experimental $(M d=1,00)$. Sobre la pregunta relacionada con la dureza del trabajo industrial en los años 20, tampoco hubo diferencias significativas $(z=-1,053, p>0,05)$ entre el grupo control $(M d=0,00)$ y el experimental $(M d=1,00)$. En cuanto a las preguntas vinculadas a los contenidos actitudinales, la cuestión relacionada con la influencia del trabajo en la salud mental de las personas no presentó diferencias significativas $(z=-0,655, p>0,05)$ : grupo control $(\mathrm{Md}=0,00)$ y grupo experimental $(M d=0,00)$. En la pregunta sobre la mejora que pueden causar las tecnologías a la vida diaria de las personas no se encontraron diferencias significativas $(z=-0,756, p>0,05)$ entre el grupo control $(M d=0,00)$ y el experimental $(M d=0,00)$.

Tabla 3. Cambio en el grado de conocimiento de contenidos en ambos grupos

\begin{tabular}{|l|c|c|c|}
\hline \multicolumn{1}{|c|}{ Variable } & Significatividad & Pre-test (Md) & Post-test (Md) \\
\hline Conocimiento de la producción en cadena & $p>0,05$ & 2,00 & 1,00 \\
\hline Dureza del trabajo en la industria años 20 & $p>0,05$ & 0,00 & 1,00 \\
\hline Influencia del trabajo en la salud mental & $p>0,05$ & 0,00 & 1,00 \\
\hline Mejora de la tecnología a la vida diaria & $p>0,05$ & 0,00 & 0,00 \\
\hline
\end{tabular}

Fuente: elaboración propia. 


\section{DISERTACIONES}

AVANCES

Por tanto, tras haber analizados los datos, no se encontró que el empleo de recursos audiovisuales en el aula sea significativo para el aprendizaje de contenidos, más concretamente, conceptuales y actitudinales.

\section{Hipótesis 3}

En la tercera de estas hipótesis se pretendía encontrar que la utilidad que el alumnado otorga a las películas como recurso didáctico aumente, respecto a su valoración previa, una vez se ha empleado una película con tal objetivo en el aula. Para ello, se concretaron cuatro utilidades diferentes de los recursos audiovisuales: "ayudar a comprender los conceptos de los contenidos", "ayudar a relacionar los contenidos de varias asignaturas", "ayudar a relacionarlos con la vida cotidiana" y "generar interés sobre los contenidos de clase".

Para comprobar esta hipótesis se creó un índice agregado sobre la utilidad de las películas compuesto por las cuatro utilidades citadas. Además, se realizó la prueba $U$ de Mann-Withney, con el índice agregado sobre la utilidad de las películas (de los datos de la encuesta) y la variable Grupo para conocer si existían diferencias significativas entre ambas clases de la sección bilingüe. Así, las diferencias no fueron significativas $(z=-0,098, p>0,05)$ entre los grupos respecto a la utilidad de las películas.

Con el objetivo de comprobar la hipótesis se realizó la prueba de Wilcoxon. Teniendo en cuenta que para la medición se empleó una escala Likert donde 1 indicaba 'Mucho' y 5 'Nada'. A tenor de los resultados ( $z=-2,208$, $p<0,05)$ se puede afirmar que hubo diferencias significativas sobre la utilidad didáctica de las películas antes del visionado $(M d=2,12)$ y después $(M d=1,75)$. Por tanto, se comprobó que hay una relación causal entre el empleo de una película y la mayor utilidad que el alumnado percibe que tienen las películas para lograr objetivos didácticos.

Tabla 4. Utilidad didáctica de las películas

\begin{tabular}{|l|c|c|c|}
\hline \multicolumn{1}{|c|}{ Variable } & Significatividad & Control (Md) & Experimental (Md) \\
\hline Utilidad didáctica de las películas & $\mathrm{p}<0,05$ & 2,12 & 1,75 \\
\hline
\end{tabular}

Fuente: elaboración propia.

En cuanto a cada una de las variables referidas a cada utilidad, la dispersión de los datos en todas las variables fue mayor en el pre-test (Rango=3) que en el post-test (Rango=2), incluyendo el índice agregado sobre utilidad, al ser en el pre-test (Rango $=2,25)$ y en el post-test (Rango $=1,25)$. 


\section{DISERTACIONES}

AVANCES

Tecnologías y métodos computacionales para la investigación en ciencias sociales y comunicación

ISSN: 1856-9536

Doi: https://doi.org/10.12804/revistas.urosario.edu.co/disertaciones/a.7310

Volumen 13, Número 1 / Enero-junio 2020

Versión PDF para imprimir desde

http://revistas.urosario.edu.co/index.php/disertaciones

Tabla 5. Dispersión de los datos sobre las utilidades didácticas de las películas

\begin{tabular}{|l|c|c|}
\hline \multicolumn{1}{|c|}{ Variable } & Rango pre-test & Rango post-test \\
\hline Ayudar a comprender conceptos de los contenidos & 3,00 & 2,00 \\
\hline Ayudar a relacionar conceptos de varias asignaturas & 3,00 & 2,00 \\
\hline Ayudar a relacionar contenidos de la vida cotidiana & 3,00 & 2,00 \\
\hline Generar interés sobre los contenidos de clase & 3,00 & 2,00 \\
\hline Utilidad didáctica general de las películas & 2,25 & 1,25 \\
\hline
\end{tabular}

Fuente: elaboración propia.

Estos datos indican que el grupo experimental tenía percepciones menos dispersas sobre la utilidad didáctica de las películas que días antes, tanto en cada una de las utilidades como en la utilidad general de las películas.

\section{Discusión}

Gracias al trabajo experimental realizado con el alumnado del centro educativo se han podido conocer algunos indicios sobre qué relación puede haber entre los contenidos y recursos didácticos y el nivel de competencia de Aprender a aprender.

Así, los datos obtenidos muestran que podría haber una relación entre la percepción positiva de la utilidad de los contenidos de clase medido en dos ejes temporales: en el presente y en el futuro, y la frecuencia con la que el alumnado reflexiona sobre si ha aprendido o por qué ha aprendido o no lo ha logrado.

También se ha identificado una posible relación entre el aumento de la utilidad didáctica que el alumnado cree que pueden tener los recursos audiovisuales y su empleo en clase, lo que puede influir en un potencial desarrollo de su aprendizaje autónomo.

No se ha apreciado relación entre la capacidad para aprender de manera autónoma y la búsqueda de información por estos productos audiovisuales, ni tampoco diferencias significativas entre los estudiantes en cuanto al nivel de aprendizaje autónomo y la frecuencia en buscar información por los formatos audiovisuales, si bien sí hay diferencias por sexos en cuanto al tipo de formatos sobre el que buscan información. En el caso de las alumnas es más frecuente que indaguen más sobre contenidos de la televisión y el cine, mientras que los alumnos son más propensos a realizarlo utilizando los videojuegos.

La frecuencia en la búsqueda de información debido al consumo de productos audiovisuales se encuentra en un nivel medio. No obstante, teniendo en cuenta que como indican los datos, los motivos por los que el alumnado busca información más frecuentemente por estos productos es porque les gusta el contenido y, por otro lado, aquellos que consideran los contenidos de clase más entretenidos reflexionan más sobre su aprendizaje, se podría

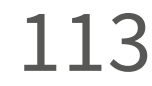




\section{DISERTACIONES}

AVANCES

considerar que hay más posibilidades de potenciar la búsqueda autónoma de información relacionada con recursos didácticos audiovisuales en la medida en que el contenido de estos les resulte entretenido. Es decir, que el potencial didáctico de lo audiovisual y la curiosidad de los estudiantes se relacionen gracias al contenido de estos recursos, y ello potencie y facilite su capacidad para Aprender a aprender.

No obstante, la mejora del alumnado en cuanto a su aprendizaje autónomo a través de recursos audiovisuales puede tener diferentes problemas, algunos citados antes, como la dificultad del centro educativo para obtener los materiales didácticos y audiovisuales necesarios para llevar a cabo esta estrategia, o la falta de formación del profesorado en este ámbito. Carencias que en ocasiones sufren los centros, a pesar de que "es otra competencia ineludible de la administración educativa, ya que es inviable el desarrollo de innovaciones curriculares que no cuenten con los mínimos soportes didácticos de apoyo" (Aguaded, 2005, p. 53). Para contrarrestar esta situación, las instituciones gubernamentales (autonómicas y estatales) de España han llevado a cabo acuerdos para realizar inversiones públicas, como el convenio marco de colaboración con Red.es para lograr una mejor conexión a Internet en los centros educativos. Sin embargo, a pesar de haberse aprobado en 2015, ${ }^{7}$ aún no se ha comenzado a llevar a cabo al no haber finalizado la adjudicación empresarial de estos proyectos en algunas Comunidades Autónomas.

En cualquier caso, para trabajar las competencias Aprender a aprender y Competencia digital mediante recursos audiovisuales, quizá los requisitos fundamentales - una vez se dispone de una mínima cantidad y calidad de medios y materiales-son saber emplear eficazmente estos recursos y la proactividad del alumno. El uso de recursos audiovisuales requiere de una participación activa del estudiante, de su interacción con los medios y materiales y con el resto de compañeros.

\section{Conclusiones}

En la investigación se trató de descubrir si el uso de recursos audiovisuales facilita el aprendizaje de contenidos conceptuales y actitudinales y averiguar si el empleo de recursos audiovisuales en el aula mejora la percepción del alumnado sobre la utilidad didáctica de estos recursos y, por tanto, mejora la capacidad para aprender de manera autónoma.

Así, estos objetivos se han cumplido, si bien se han obtenido diferentes resultados. Por una parte, a tenor de los datos analizados, el alumnado sí percibe que los recursos audiovisuales pueden ser mucho más útiles para su aprendizaje tras haber sido utilizados en el aula, lo que señala que para que sean eficaces se requiere de una asimilación previa del alumnado sobre la capacidad de lo audiovisual como elemento didáctico. De este modo, podrían lograr este metaconocimiento sobre el proceso de aprendizaje al ser consciente de su utilidad; es decir,

7 El 27 de marzo de 2015 se aprobó en Consejo de Ministros un acuerdo, que autorizaba a los Ministerios de Industria, Energía y Turismo, de Educación, Ciencia y Deporte y de Economía y Competitividad la suscripción de un convenio marco de colaboración con Red.es para la extensión del acceso a la banda ancha rápida a los centros docentes españoles, haciendo especial hincapié en aquellos de zonas rurales y remotas. Un convenio de colaboración en el que participaron las ccAA de La Rioja, Castilla-La Mancha, Andalucía, Región de Murcia, Canarias, Galicia y Principado de Asturias.

\section{4}




\section{DISERTACIONES}

AVANCES

se lograría un andamiaje sobre los conocimientos y logros y el proceso seguido, siguiendo las teorías pedagógicas constructivas de Piaget (1987) mediante los procesos de asimilación y acomodación.

Sin embargo, en esta investigación exploratoria no se ha podido comprobar que el uso de recursos audiovisuales en el aula ofrezca mejores resultados que el exclusivo desarrollo de una clase ordinaria, si bien sí mejora el aprendizaje de los contenidos curriculares por el alumnado.

También se ha podido comprobar, ante las dudas y preguntas formuladas por el alumnado encuestado, el bajo o nulo hábito que tienen en cuanto a reflexionar u opinar sobre su propio aprendizaje, al igual que sobre actividades de auto-evaluación, lo que puede influir a su vez en un menor potencial de mejora sobre la competencia Aprender a aprender. Ello es muy relevante si se considera que esta capacidad permite a los estudiantes tener más opciones de adaptarse a las circunstancias a lo largo de la vida (life-long learning), saber lidiar con contextos desconocidos y emplear distintas estrategias para conseguir sus objetivos.

El nivel mostrado por el alumnado tampoco parece ser especialmente elevado en el ámbito de la alfabetización mediática, ya que a pesar de que en algunas ocasiones se logra discernir el mensaje subyacente, en otras no ocurre lo mismo, como la crítica que la película Tiempos Modernos (1936) realizaba sobre el desarrollo y las nuevas tecnologías.

Este estudio exploratorio puede abrir nuevas vías de investigación sobre la competencia Aprender a aprender y los productos audiovisuales en la enseñanza: una relación apenas explorada, especialmente en la literatura científica en España. No obstante, existen algunas excepciones, como la investigación experimental realizada por Díaz Membrives (2017) en nivel educativo universitario, en la que trataba de evaluar la efectividad de una intervención docente basada en el visionado de secuencias de películas comerciales con el fin de mejorar el aprendizaje de conocimientos de los estudiantes. En cualquier caso, este trabajo de campo debería ser continuado y ampliado con una muestra mayor para que los datos obtenidos pudieran tener mayor fiabilidad, para así poder ser extrapolados y, de igual modo, ratificar las conclusiones extraídas en la presente investigación.

Entre las investigaciones de referencia que cabría citar para desarrollar nuevas investigaciones en este campo, destaca la realizada por Barak, Ashkar y Dori (2011), especialmente por la estrecha relación con aspectos fundamentales del aprendizaje autónomo -en el que se centró la presente investigación-como la motivación para aprender, el entretenimiento y disfrute generado, la relación de los contenidos con la vida diaria o su importancia en el futuro, así como la desarrollada por Marsh, Butler y Umanath (2012), debido a la materia educativa tratada, Historia, y la relevancia que otorgan a la labor del mediador para evitar los posibles errores de compresión o inexactitudes causados por los recursos audiovisuales.

\section{Referencias}

1. Aebli, H. (1991). Factores de la enseñanza que favorecen el aprendizaje autónomo. Madrid: Narcea.

2. Barak, M., Ashkar, T., \& Dori, Y.J. (2011). Learning science via animated movies: Its effect on students' thinking and motivation. Computers \& Education, 56(3), 839-846. Recuperado de https://www.sciencedirect.com/ science/article/pii/S0360131510003106?via\%3Dihub

3. Barnett, M., \& Kafka, A. (2007). Using Science Fiction Movie Scenes to Support Critical Analysis of Science. Journal of College Science Teaching, 31(4), 31-35. 


\section{DISERTACIONES}

AVANCES

Tecnologías y métodos computacionales para la investigación en ciencias sociales y comunicación

4. Agencia Estatal Boletín Oficial del Estado (BOE) (2015). Orden ECD/65/2015. BOE, 25,6986-7003. Recuperado de https://www.boe.es/buscar/doc.php?id=BOE-A-2015-738

5. Bouman, M. (1999). Collaboration for Pro-social Change: The Turtle and the Peacock. The EntretainmentEducation Strategy on Television. Holanda: Thesis Wageningen Agricultural University.

6. Bovill, M., \& Livingstone, S. M. (2006). Bedroom culture and the privatization of media use En Bovill, M., \& Livingstone, S.M. (eds). Children and Their Changing Media Environment: a European Comparative Study (pp. 179-200). Nueva Jersey: Lawrence Erlbaum Associates.

7. Chaplin, C. (director y productor). (1936). Tiempos modernos. [Película]. Estados Unidos: United Artists.

8. Claxton, G. (2005). Aprendiendo a aprender: objetivo clave en el currículum del siglo XXI. Cuadernos de Información y Comunicación (cIC), (10), 259-265. Recuperado de https://revistas.ucm.es/index.php/CIYC/ article/download/CIYC0505110259A/7301

9. Delors, J. (1996). Los cuatro pilares de la educación. En La educación encierra un tesoro. Informe a la unEsCo de la Comisión internacional sobre la educación para el siglo. Recuperado de http://uom.uib.cat/digitalAssets/221/221918_9.pdf

10. Díaz Membrives, M. (2017). Efectividad de la proyección de secuencias cinematográficas en el Grado de Enfermería. (Tesis doctoral). Universitat de Barcelona, Barcelona. Recuperado de http://diposit.ub.edu/ dspace/bitstream/2445/113430/1/MDM_TESIS.pdf

11. Faros para la Sociedad de la Información (2005). Declaración de Alejandría sobre la alfabetización informacional y el aprendizaje a lo largo de la vida. Recuperado de https://www.ifla.org/node/7275

12. Fedele, M., \& García-Muñoz, N. (2010). El consumo adolescente de la ficción seriada. Vivat Academia, (111). Recuperado de http://www.vivatacademia.net/index.php/vivat/article/view/204

13. Flavell, J. H. (1982). La psicología evolutiva de Jean Piaget. Barcelona: Paidós.

14. García, M. D., \& Benítez, M. V. (1998). Formación de profesores en alfabetización audiovisual. Comunicar, 11, 202-207. https://doi.org/10.3916/C11-1998-31

15. Gilliam, A., \& Seltzer, R. (1989). The Efficacy of Educational Movies on Aids Knowledge and Attitudes among College Students. Journal of American College Health, 37(6), 261-265. https://doi.org/10.1080/07 448481.1989 .9937492

16. Howe, N., \& Strauss, W. (2000). Millennials Rising: The Next Great Generation. Nueva York: Vintage Books.

17. Igartua, J. J. (2006). Métodos cuantitativos de investigación en comunicación. Barcelona: Bosch.

18. Jefatura del Estado. (3 de mayo de 2006). Ley Orgánica de Educación [Ley 2/2006]. DO: BOE, Madrid, España, 4 de mayo de 2006. Recuperado de http://www.boe.es/buscar/act.php?id=BOE-A-2006-7899\&tn=1\&p= 20150729\#a102

19. Kaplún, M. (2002). Una pedagogía de la Comunicación (el comunicador popular). La Habana: Editorial Caminos. Recuperado de http://perio.unlp.edu.ar/catedras/system/files/kaplun-el_comunicador_popular_0.pdf

20. López, N., González, A., \& Medina, E. (2011). Jóvenes y televisión en 2010: Un cambio de hábitos. ZER Revista de Estudios de Comunicación, 30, 97-113. Recuperado de https://www.ehu.eus/ojs/index.php/Zer/ article/view/4793/4659 
21. Mallas, S. (1977). Técnicas y recursos audiovisuales: teoría y práctica. Barcelona: OIKOS-TAU.

22. Marcos, M. (2010). Mediación y alfabetización mediática: una propuesta de actuación para reducir el efecto negativo de la violencia audiovisual (tesis doctoral). Universidad de Salamanca, Salamanca, España.

23. Marín, C. (2006). Periodismo audiovisual: información, entretenimiento y tecnologías multimedia. Barcelona: Gedisa.

24. Marsh, E., Butler, A., \& Umanath, S. (2012). Positive and Negative Effects of Monitoring Popular Films for Historical Inaccuracies. Applied Cognitive Psychology, 26(4), 556-567. https://doi.org/10.1002/acp.2827

25. Martín, E., \& Moreno, A. (2007). Competencia para aprender a aprender. Madrid: Alianza Editorial.

26. Monereo, C. (coord.) (2001). Ser estratégico y autónomo aprendiendo. Unidades didácticas de enseñanza estratégica para la Eso. Barcelona: Graó.

27. Organización para la Cooperación y el Desarrollo Económicos (OCDE). Definition and Selection of Competencies (DeSeCo). (Sin fecha). Recuperado de https://www.oecd.org/education/skills-beyond-school/definitionandselectionofcompetenciesdeseco.htm

28. Piaget, J. (1987). Psicología y pedagogía. Barcelona: Ariel.

29. Prensky, M. (2001). Digital Natives, Digital Immigrants. On the Horizon, 9(5). Recuperado de http://www.marcprensky.com/writing/Prensky\%20-\%20Digital\%20Natives,\%20Digital\%20Immigrants\%20-\%20Part1.pdf

30. Quin, R., \& Sánchez Martínez, M. (1999). Aprender a mirar: el lugar de los textos visuales en el currículum escolar. Comunicar, 12, 131-139. https://doi.org/10.3916/C12-1999-21

31. Rivière, A. (1988). La psicología de Vygotski. Madrid: Visor Distribuciones.

32. Singhal, A., \& Rogers, E. M. (1999). Entertainment-education: A Communication Strategy for Social Change. Mahwah, NJ: Lawrence Erlbaum.

33. UnESCO (1982). Declaración de Grünwald sobre la educación relative a los medios de comunicación. Recuperado de http://www.unesco.org/education/pdf/MEDIA_S.PDF

34. Vigotski, L. S. (1989). El desarrollo de los procesos psicológicos superiores. Barcelona: Crítica. 